\title{
CONOCIMIENTOS, ACTITUDES Y PRÁCTICAS RELACIONADOS CON LACTANCIA MATERNA EN MUJERES EN EDAD FÉRTIL EN UNA POBLACIÓN VULNERABLE.
}

\section{KNOWLEDGE, ATTITUDES AND PRACTICES RELATED TO BREAST-FEEDING IN WOMEN IN REPRODUCTIVE AGE IN A VULNERABLE POPULATION.}

\author{
Edna Magaly Gamboa D. $(1,2,3,4)$, Nahyr López B. $(1,3,4)$ \\ Gloria Esperanza Prada G. (2,3), Kelly Yanitze Gallo P. (2)
}

(1) Departamento de Salud Pública, Escuela de Medicina, Universidad Industrial de Santander (UIS)

(2) Escuela de Nutrición y Dietética, UIS.

(3) Grupo de Investigación Observatorio Epidemiológico de Enfermedades Cardiovasculares (OEECV).

(4) Grupo de Investigación en Demografía, Salud Pública y Sistemas de Salud (GUINDESS).

Este trabajo fue recibido el 8 de Octubre de 2007 y aceptado para ser publicado el 3 de Marzo de 2008.

Dirigir la correspondencia a:

Profesora

Edna Magaly Gamboa Delgado

Carrera 32 \# 29-31 piso 3

Departamento de Salud Pública

Facultad de Salud, UIS

Bucaramanga. Colombia.

Teléfono; (57-7) 6454726

E-Mail: magalygamboa@yahoo.com 


\begin{abstract}
Objective: to identify the knowledge, attitudes and practical related with the breast feeding (BF) in women in reproductive age residents of the establishment the Marías of the Municipality of Girón in the year 2005. Subjects and method: a descriptive cross sectional with 96 women in fertile age of the establishment the Marías, Girón, 2005 was carried out. A survey, type Knowledge, Attitudes, Practices (KAP) was applied. The data were fingered and validated in Epi - Info 6,04 and analyzed in Stata 8,2. To analyze the continuous variables, central tendency measures were used and of variability; the categorical variables were expressed as proportions. Results: $78,1 \%$ of the participants had had children. $13 \%$ only had knowledge on the correct form of breastfeeding. $49 \%$ didn't know the methods of conservation of the maternal milk. $69,8 \%$ of the participants has support attitude when you see to a family one suckling. The time average of maternal nursing was 5,7 months. The food with which more frecuently began the complementary feeding was the broth $(88 \%)$. Conclusions: A considerable proportion of women of the study gave BF less than six months, they began the complementary feeding before the 6 months and that the BF suspended when the son suffered some illness.
\end{abstract}

Key words: breast feeding; knowledge; attitudes; practices; vulnerable population.

\title{
INTRODUCCIÓN
}

La leche materna constituye el mejor alimento que puede darse en forma exclusiva a un niño o niña hasta los seis meses de edad y con otros alimentos hasta los dos años (1). La Organización Mundial de la Salud (OMS) y el Fondo de Naciones Unidas para la infancia (UNICEF) han considerado por décadas a la lactancia materna (LM) fundamental en la alimentación humana y la han promovido mediante diversas iniciativas globales (2).

A nivel internacional existen diversas instituciones que apoyan y promueven la lactancia materna, como la UNICEF, la IBFAN (International Baby-Food Action Network) y la WABA (World Alliance for Breastfeeding Action) (3, 4). En Colombia, el fomento de la lactancia materna se ha desarrollado mediante la realización de distintos programas, algunos son: la estrategia IIAMI (Iniciativa Instituciones Amigas de la Mujer y la Infancia) y la realización de la Semana Mundial de Lactancia Materna que se celebra cada año durante la primera semana de agosto y es apoyada por la WABA.

La leche materna le proporciona al niño todos los nutrientes que necesita durante los primeros seis meses de vida en forma complementaria hasta los dos años, pr tanto es importante que el inicio de otros alimentos sea en un momento oportuno y no tempranamente. La lactancia materna le aporta al lactante los nutrientes necesarios para su crecimiento y desarrollo (5) y además lo protege de enfermedades (68); en la madre disminuye la mortalidad materna, el riesgo de cáncer de mama, fomenta el amor con su hijo; en la familia, contribuye a la integración y a la economía familiar (9). La lactancia materna ofrece además beneficios sociales, como la reducción de las tasas de mortalidad y morbilidad infantil, la contribución a una población infantil sana y favorece la no violencia (10). 
Colombia se ha interesado desde hace varios años en promover la práctica de LM. Ya se empiezan a ver algunos resultados; la encuesta nacional de la situación nutricional realizada en el año 2005, mostró que en los últimos 10 años el porcentaje de mujeres que inicia la LM, aumentó en 2,6\%, la duración total de LM aumentó en 3,6 meses y la Lactancia Materna Exclusiva (LME) en 1,7 meses. En ese año la duración total promedio fue de 14,9 meses y de LME de 2,2 meses (11).

En una población desplazada (desastre social producto del conflicto armado interno en el cual la población se ve obligada a migrar de las zonas rurales a las urbanas) el tiempo de lactancia es menor (12, 13). Esta situación amerita profundizar los estudios hacia las razones que motivan o no la LM en esta población.

Los estudios tipo Conocimientos, Actitudes y Prácticas (CAP), han sido utilizados en diversos temas de salud y se consideran como la base fundamental de los diagnósticos para ofrecer información a Instituciones u Organizaciones responsables de la creación, ejecución y evaluación de programas de promoción de la salud y nutrición.

El propósito de esta investigación fue elaborar un diagnóstico sobre la situación de la LM en una comunidad desplazada, que además fue la más afectada por la avalancha invernal del año 2005 ocurrida en Girón, Santander, con el fin de establecer estrategias de intervención al respecto.

El objetivo de este estudio fue identificar los conocimientos, actitudes y prácticas relacionados con la lactancia materna en mujeres en edad fértil de la población vulnerable estudiada.

\section{SUJETOS Y MÉTODO}

Un estudio de corte transversal descriptivo fue llevado a cabo durante el segundo semestre del año 2005 en el sector Las Marías del municipio de Girón, Santander (asentamiento conformado por población desplazada por la violencia y damnificada por la ola invernal). Teniendo en cuenta que para el segundo semestre de 2005 existían, aproximadamente, 122 mujeres en ese asentamiento, se incluyeron las de 14 a 45 años de edad, para lo cual se realizaron visitas a todas las casas de una en una y se encuestó a todas las mujeres que cumplieron con este criterio.

Se utilizó el muestreo por conveniencia. Participaron en el estudio 96 mujeres en edad fértil. Se excluyeron del estudio a las mujeres que presentaron incapacidad física o mental para contestar una encuesta.

A las participantes del estudio se les aplicó una encuesta estructurada, mediante entrevista directa, efectuada por estudiantes de últimos semestres de la carrera de Nutrición y Dietética de la Universidad Industrial de Santander, previamente entrenados. El formato de la encuesta contenía cuatro secciones correspondientes a: datos sociodemográficos y económicos, conocimientos, actitudes y prácticas 
relacionados con la lactancia materna. Los aspectos relacionados con los conocimientos y las actitudes frente a la lactancia materna, fueron evaluados en todas las mujeres participantes del estudio, mientras los referentes a Prácticas sólo fueron indagados en las mujeres que habían tenido hijos.

Se evaluaron variables sociodemográficas (edad, ocupación, estado civil, escolaridad, número de hijos) económicas (ingresos), y otras relacionadas con los conocimientos, las actitudes y las prácticas respecto a la lactancia materna. Se consideró como práctica de lactancia materna exclusiva aquella en la cual las madres ofrecieron a sus hijos únicamente lactancia materna durante los seis primeros meses de vida, sin agregar ningún otro tipo de alimento.

\section{Análisis de datos}

La información fue digitada por duplicado y validada en el programa Epi Info v 6,04d. Se generaron los resultados en el programa Stata v 8,2 para obtener estadísticas univariadas, en las que se presentan las variables continuas con sus medidas de tendencia central y sus correspondientes medidas de dispersión y las variables categóricas fueron presentadas como frecuencias. Se compararon los conocimientos y creencias con las prácticas de los tiempos de inicio y duración de la LM y LME, y el tiempo de inicio de alimentación complementaria, utilizando la prueba t de Student. Se realizó un análisis bivariado entre los factores socioeconómicos y la práctica de lactancia materna exclusiva, calculando las razones de riesgo relativas (OR) y sus Intervalos de confianza del 95\% (IC 95\%).

Todas las participantes firmaron el consentimiento informado. De acuerdo con la Resolución $\mathrm{N}^{\mathrm{o}} 08430$ de 1993, del Ministerio de Salud de Colombia, este trabajo se clasifica como investigación sin riesgo para los participantes. Las mujeres encuestadas fueron identificadas con códigos respetando así los principios fundamentales de la ética: autonomía, beneficencia, no maleficencia y justicia.

\section{RESULTADOS}

\section{Características sociodemográficas y económicas}

Un total de 96 mujeres en edad fértil fueron encuestadas. El 11,5\% de las participantes procedía del área rural. El 24,0\% de las encuestadas tenía una edad menor o igual a 20 años. La mitad de las participantes del estudio se encontraba en unión libre. Respecto al nivel de escolaridad, se encontró un $52,1 \%$ de mujeres que había cursado y aprobado cinco o menos años de educación formal. El 78,1\% de las participantes había tenido hijos; el 38,1\% de ellas, había tenido un hijo, 22,6\% dos hijos, 6,0\% 3 hijos y 1,2\% 4 hijos. De las mujeres que habían tenido hijos, un 63,5\% convivía con el padre de éstos. La figura 1 presenta el tiempo promedio de duración de la LM y LME, según la edad de la madre. Las características generales de la muestra estudiada se presentan en la tabla 1. 
Tiempo promedio de duración de la LM y LME, según la edad de la madre

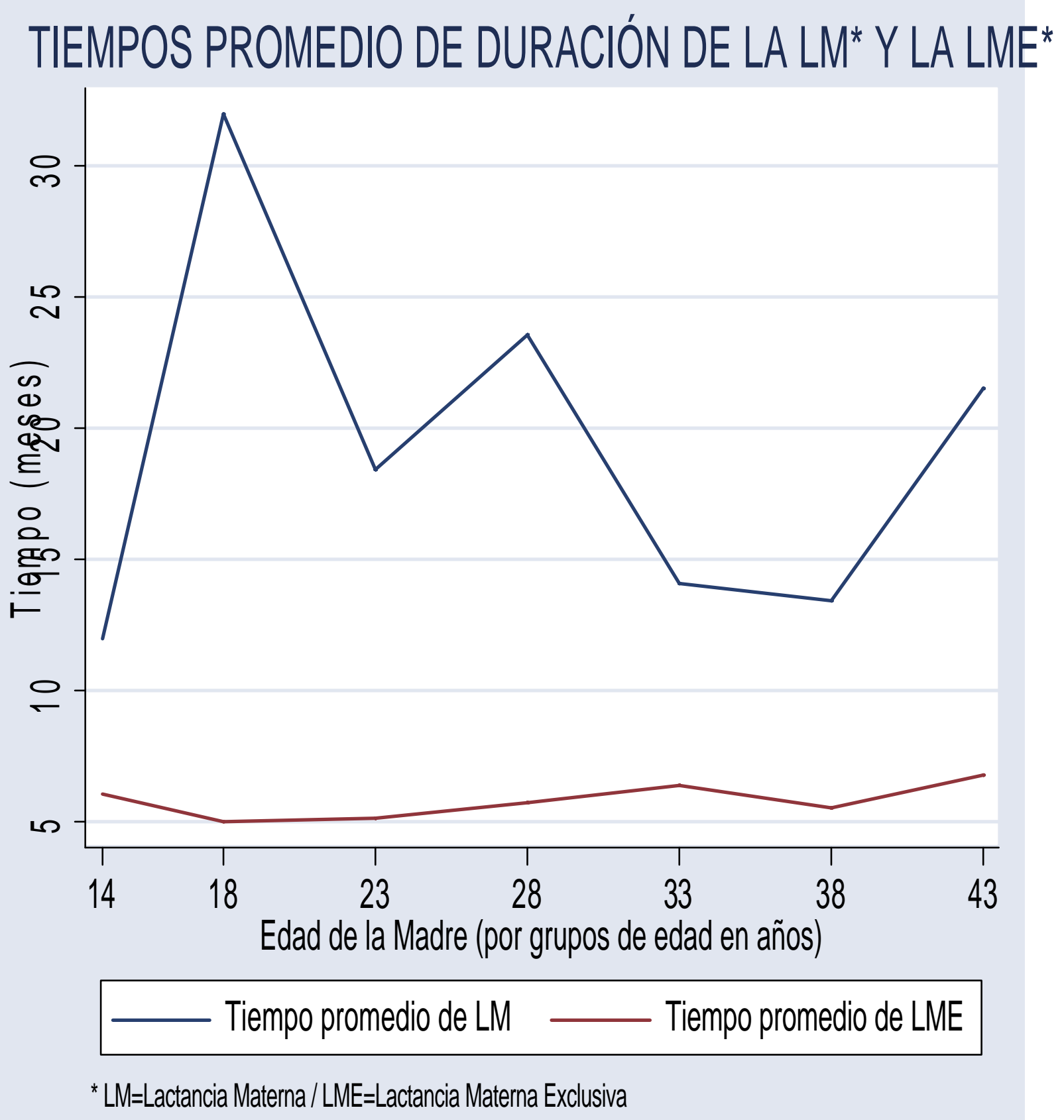

* LME: Lactancia Materna Exclusiva / LM: Lactancia Materna 
TABLA 1

Características generales de las mujeres estudiadas (n=96)

\begin{tabular}{|c|c|c|}
\hline Características & $\begin{array}{c}\text { Promedio o } \\
\text { Número }\end{array}$ & $\begin{array}{l}\text { IC }(95 \%) \text { o } \\
\text { Porcentaje }\end{array}$ \\
\hline Edad $^{\mathrm{a}}$ (años) & 27,4 & $(25,6 ; 29,1)$ \\
\hline \multicolumn{3}{|l|}{ Ocupación $^{b}$} \\
\hline Ama de casa & 60 & 62,5 \\
\hline Estudiante & 12 & 12,5 \\
\hline Empleada & 9 & 9,3 \\
\hline Independiente & 8 & 8,3 \\
\hline Desempleada & 2 & 2,0 \\
\hline Jubilada & 1 & 1,0 \\
\hline Otras & 4 & 4,1 \\
\hline \multicolumn{3}{|l|}{ Estado Civil $^{\mathrm{b}}$} \\
\hline Unión Libre & 47 & 48,9 \\
\hline Soltera & 25 & 26,0 \\
\hline Casada & 15 & 15,6 \\
\hline Separada & 5 & 5,2 \\
\hline Divorciada & 2 & 2,0 \\
\hline Viuda & 2 & 2,0 \\
\hline Nivel de Escolaridad $^{\text {a }}$ (años) & 6,9 & $(5,0 ; 8,7)$ \\
\hline Número de hijos ${ }^{a}$ & 2,9 & $(2,3 ; 3,5)$ \\
\hline \multicolumn{3}{|l|}{ Ingresos Económicos ${ }^{b}$} \\
\hline Menos de 1 SMMLV & 75 & 78,1 \\
\hline Entre 1 y 2 SMMLV & 21 & 21,8 \\
\hline
\end{tabular}

${ }^{\mathrm{a}}$ : promedio e intervalo de confianza del $95 \%$

${ }^{\mathrm{b}}$ : número y porcentaje 


\section{Conocimientos y Creencias respecto a la Lactancia Materna}

El 24,0\% de las participantes del estudio manifestó que esperaría dos horas o más, después del nacimiento, para iniciar la lactancia. Para el 97,9\% de las mujeres, dar Lactancia Materna trae beneficios para su hijo, entre ellos, ayudar al crecimiento del bebé (44,7\%), evitar enfermedades (43,6\%), favorecer el vínculo afectivo $(7,4 \%)$ y otros $(4,3 \%)$.

La mayoría de participantes conocen que la lactancia materna tiene un beneficio para ellas $(53,1 \%)$. Las encuestadas consideran que el mayor beneficio es evitar el cáncer de mama $(29,4 \%)$, favorecer el vínculo afectivo madre-hijo $(25,5 \%)$, descongestionar hs mamas $(21,6 \%)$ y ahorrar dinero $(11,8 \%)$. Para el $63,5 \%$ de las mujeres encuestadas, el calostro es necesario para la alimentación del bebé, frente a un $6,3 \%$ que considera lo contrario.

Las mujeres consideran que existen algunas prácticas por parte de la madre que alteran la composición y características de la leche materna, ellas son: consumo de alimentos picantes (29,3\%), bebidas alcohólicas $(19,5 \%)$, medicamentos $(17,1 \%)$ y alimentos ácidos $(14,6 \%)$. En cuanto a los conocimientos sobre la conservación de la leche materna, un 41,7\% considera que este alimento es susceptible a la contaminación.

Frente a los métodos de conservación de la leche materna, un 49,0\% de las participantes no los conocía. Acerca del lugar indicado para conservar la leche materna extraída el 69,4\% cree que se debe conservar en el refrigerador, el $17,6 \%$ a temperatura ambiente y el $12,9 \%$ en el congelador.

De los tipos de leche, diferentes a la leche materna, las mujeres enc uestadas creen que la leche de vaca sería la mejor opción en un $80,2 \%$ de los casos, mientras el 19,8\% seleccionó a las fórmulas lácteas infantiles.

Respecto al conocimiento que tenían las mujeres encuestadas sobre la manera adecuada de amamantar en situaciones especiales, sólo el 13,5\% afirmó que sí tenía conocimientos al respecto y de éstas, el 38,5\% conocía las formas de amamantar si la madre tiene gemelos, esta misma proporción expresó que tenía este conocimiento en caso de amamantar a niños de edades diferentes y un 23,1\% tenía esta información si se trataba de amamantamiento en niños prematuros.

Se evaluaron los conocimientos sobre el método correcto de extracción de leche materna, y el 42,7\% afirmó que no conocía el método.

Sólo el 25.0\% de las madres sabían como tratar las dificultades presentadas durante el amamantamiento, el 33,3\% tenía conocimientos sobre tratamiento en caso de mastitis o infección de las mamas, el 25,0\% en caso de pezones congestionados, o dolor en los pezones y grietas y el 8,3\% en caso de pezones invertidos o insuficiente producción de leche. 
El $27 \%$ de las participantes considera que la edad ideal de inicio de la ablactación es a los seis meses o más. Un $89,6 \%$ considera que la alimentación complementaria debe iniciar con los jugos e igual proporción para las compotas, el 81,3\% cree que deben ser los caldos, el 80,2\% las sopas, el 77,1\% las cremas de verduras, el 63,5\% los purés, el 57,3\% los cereales, el 56,3\% el huevo, y el 44,8\% las carnes.

El $85,4 \%$ de las participantes no conoce acerca de las normas que promueven y/o protegen la lactancia materna.

A las mujeres del estudio se les indagó sobre los aspectos que consideraban más importantes frente a la compra de fórmulas lácteas por parte de los padres; el 70,8\% de las participantes considera que los hace adquirir una mayor responsabilidad frente a los hijos, un $56,3 \%$ percibe que este aspecto puede favorecer un mayor vínculo afectivo padre-hijo y el 43,8\% cree que favorece un mayor acercamiento padre-madre del bebé.

El 53,1\% de las encuestadas no cree que la lactancia materna puede servir como método anticonceptivo.

\section{Actitudes frente a la lactancia materna}

Frente a la responsabilidad de la compra de las fórmulas lácteas, en caso de ser necesario, el 15,6\% de las participantes expresó que debía ser del padre, un 7,3\% dijo que era responsabilidad de la madre y un $77,1 \%$ considera que debe ser una responsabilidad compartida por ambos.

Respecto a los sentimientos que experimentan las mujeres encuestadas, cuando les hablan de lactancia materna, el 57,3\% siente ternura, 16,7\% aceptación, $12,5 \%$ desinterés y un 1,0\% siente rechazo.

Se evalúo la actitud que los participantes asumen cuando tienen alguna familiar que se encuentra lactando, y se encontró una actitud de apoyo en el 69,8\% de los casos, desinterés en el 13,5\% y solidaridad en el $12,5 \%$ de las oportunidades.

Respecto a las razones que motivan la práctica de LM también se encontró un 97,9\% de mujeres que se sienten motivadas por el hecho de ver crecer a sus hijos sanos, el 97,9\% por evitar enfermedades y alergias en el niño, el $76,0 \%$ por información recibida sobre el tema, $64,6 \%$ por el ejemplo de otros familiares y el $60,4 \%$ por la actitud positiva del esposo.

De manera contraria, se desmotivarían frente a la lactancia materna por dolor en las mamas $(38,5 \%)$, por el escaso tiempo $(28,1 \%)$, por la dedicación que requiere $(28,1 \%)$, por tener que cuidar otros hijos pequeños $(24,0 \%)$, por pena $(16,7 \%)$, por tener que realizar múltiples oficios en el hogar $(16,7 \%)$, por consejos de familiares $(15,6 \%)$, y por celos del esposo frente al nuevo bebé lactante $(10,4 \%)$. 


\section{Prácticas de la lactancia materna}

El 16\% de las mujeres que habían tenido hijos, esperó tres horas o más para iniciar la lactancia materna. El 26,7\% de las mujeres con hijos, dio lactancia materna durante menos de seis meses y un $8,0 \%$ no dio LME. La leche materna fue dada exactamente hasta los doce meses por el $20 \%$ de las madres de este estudio.

Respecto a la alimentación complementaria, el 28,0\% de las mujeres la inició antes de los seis meses, el $49,3 \%$ a los seis meses y el 22,9\% después de esta edad. Dentro de los alimentos con los cuales iniciaron la alimentación complementaria, se encontraron: caldos (88,0\%), jugos $(86,7 \%)$, compotas $(84,0 \%)$, sopas $(70,7 \%)$, crema de verduras $(62,7 \%)$, huevo $(53,3 \%)$, purés $(49,3 \%)$, cereales $(49,3 \%)$ y carnes $(45,3 \%)$.

El 81,3\% de las mujeres con hijos manifestó no usar los métodos de conservación de la leche materna.

A las personas que utilizaban métodos de conservación se les preguntó sobre el lugar donde guardaban la leche materna, encontrando un $13,3 \%$ que utiliza el congelador, $73,3 \%$ el refrigerador y el 13,3\% la conserva a temperatura ambiente. En cuanto al tipo de material de los recipientes donde conservan la leche el 20,0\% utiliza recipientes de vidrio y el 80,0\% de plástico. El tiempo máximo por el cual conservaban la leche materna fue 3,4 horas, D.E: 2,1 horas. El 40,0\% de las mujeres que manifestaron conservar la leche materna, lo hacen por 4 horas o más.

Un 42,7\% de las mujeres con hijos presentó dificultades para lactar, dentro de las cuales se encontraron: dolor y grietas en los pezones $(21,3 \%)$, mastitis $(8,0 \%)$, pezón invertido $(5,3 \%)$ y producción de leche insuficiente $(5,3 \%)$.

El 13,3\% de las mujeres con hijos suspendió la lactancia materna cuando el hijo lactante sufrió alguna enfermedad o situación específica como cólicos, diarrea, gripa, irritabilidad, falta de apetito, rechazo del seno, o ganancia lenta de peso.

Sobre si la lactancia materna representó algún tipo de impedimento o inconveniente en algunas situaciones diarias de las madres, en el $13,3 \%$ sus relaciones laborales se vieron afectadas, en el 12,0\% de los casos ocurrió alteración de sus actividades domésticas, en el 5,3\% sus relaciones interpersonales, en el 5,3\% sus relaciones sexuales y en el 2,7\% sus relaciones familiares.

El 80,0\% de las mujeres con hijos recibió apoyo durante la lactancia materna. En el 66,7\% de los casos el apoyo fue dado por el esposo, en el $60 \%$ por personal de salud, en el 58,7\% por la familia, y en el $37,3 \%$ por personas conocidas. Las mujeres encuestadas también ofrecieron apoyo a otras nodrizas, que consistió en dar un consejo en favor de la lactancia en un 57,3\%, apoyo emocional en un 28,0\%, apoyo económico en un 8,0\%, desinterés en un 5,3\% y dar una opinión en contra de la lactancia en un 1,3\%. 
En las tablas 2 y 3 se presenta la comparación entre los conocimientos/creencias de las mujeres encuestadas con hijos y las prácticas realizadas por ellas en cuanto a la lactancia materna. Los datos de la tabla 2 permiten apreciar que no se encontraron diferencias estadísticamente significativas entre lo que las mujeres encuestadas conocen o creen y lo que practicaron respecto a los tiempos de inicio y duración de la LM y LME. La tabla 3 muestra que existe una clara relación de dependencia entre los conocimientos y creencias y las prácticas acerca del aumento de producción de LM, métodos de conservación de LM y capacidad anticonceptiva de ésta.

\section{TABLA 2}

Comparación entre los conocimientos y creencias y las prácticas relacionados con el tiempo de inicio y duración de la $\mathrm{LM}^{*}$ y $\mathrm{LME}^{*}(\mathrm{n}=75)$

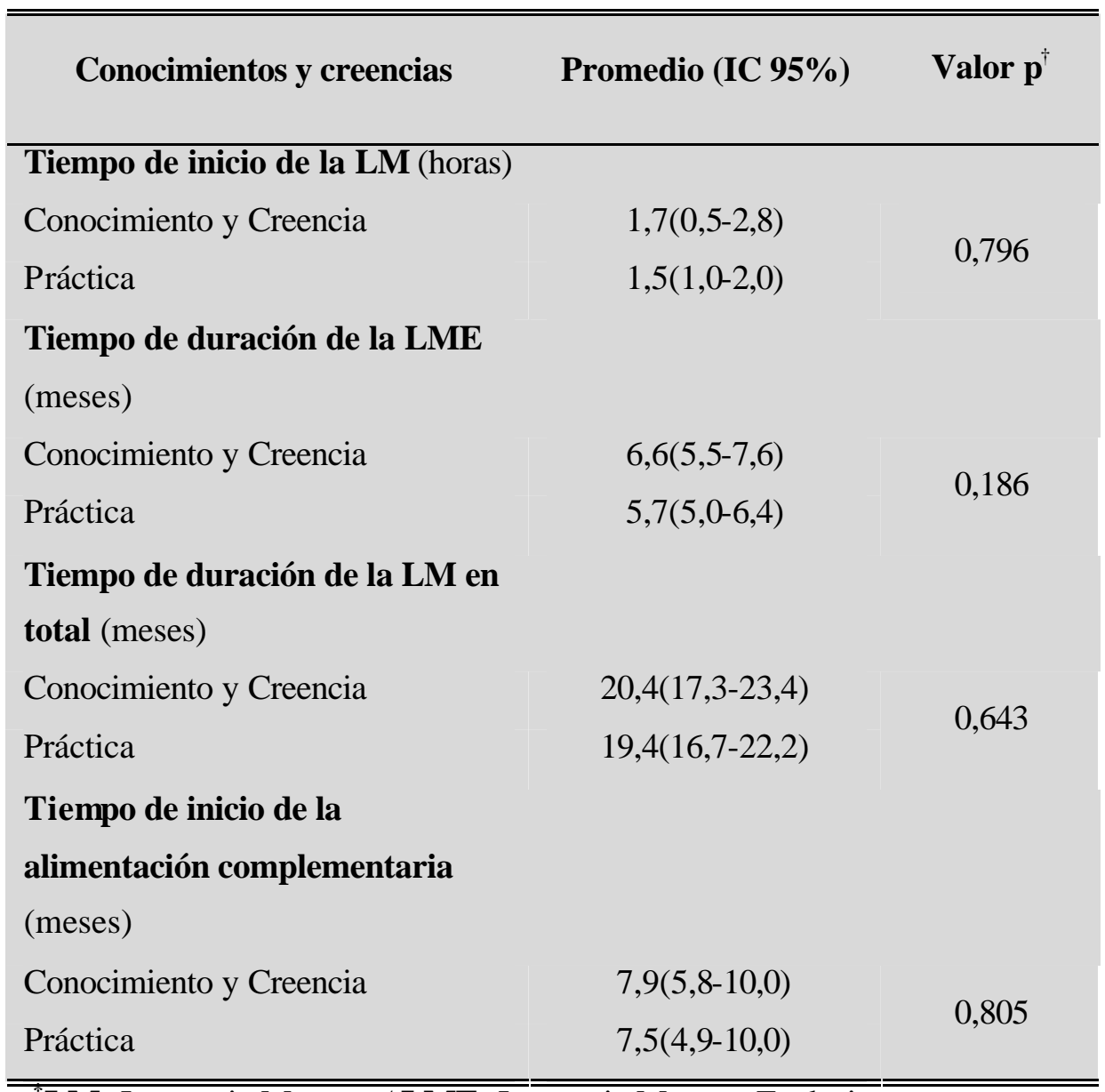

"LM: Lactancia Materna / LME: Lactancia Materna Exclusiva

${ }^{\dagger}$ : Prueba $t$ Student 
TABLA 3

Relación entre algunos conocimientos y creencias y prácticas relacionados con la $\mathbf{L M}^{*}(\mathbf{n}=\mathbf{7 5})$

\begin{tabular}{|c|c|c|c|c|}
\hline \multirow[b]{2}{*}{ Conocimiento y creencia } & & \multicolumn{2}{|c|}{ Práctica } & \multirow[t]{2}{*}{ Valor $\mathbf{p}$} \\
\hline & & $\mathrm{Si}$ & No & \\
\hline \multirow{2}{*}{$\begin{array}{l}\text { Aumento de la producción de LM a través de } \\
\text { abundantes líquidos, alimentación adecuada y } \\
\text { succión del bebé. }\end{array}$} & $\mathrm{Si}$ & $58(87,8)$ & $8(12,2)$ & \multirow[t]{2}{*}{$0.000^{\ddagger}$} \\
\hline & No & $3(33,4)$ & $6(66,6)$ & \\
\hline \multirow{2}{*}{ Métodos de conservación de leche materna. } & $\mathrm{Si}$ & $14(33,4)$ & $28(66,6)$ & \multirow{2}{*}{$0.000^{\S}$} \\
\hline & No & $0(0,0)$ & $32(100,0)$ & \\
\hline \multirow[t]{2}{*}{ LM como método anticonceptivo. } & $\mathrm{Si}$ & $7(50,0)$ & $7(50,0)$ & \multirow[t]{2}{*}{$0.000^{\ddagger}$} \\
\hline & No & $5(8,2)$ & $56(91,8)$ & \\
\hline
\end{tabular}

*LM: Lactancia Materna

: Prueba $C h i^{2}$

§: Prueba Exacta de Fisher

\section{Factores asociados a la práctica de lactancia materna}

Al establecer la asociación entre las principales características sociodemográficas de las participantes en el estudio y la práctica de LME hasta los seis meses, se encontró que tienen mayor probabilidad de dar lactancia materna exclusiva a sus hijos, aquellas mujeres que presentaron una edad de 25 años o más, las amas de casa, las que conviven con su pareja y quienes tenían 5 años o más de escolaridad; sin embargo, esta asociación no fue estadísticamente significativa. (ver tabla 4). 


\section{TABLA 4}

Factores asociados a $\mathrm{LME}^{ \pm}$hasta los seis meses $(\mathrm{n}=75)$

\begin{tabular}{lllc}
\hline \hline \multicolumn{1}{c}{ Factor } & OR & IC 95\% & Valor $\boldsymbol{p}^{\dagger}$ \\
\hline Edad: mayor o igual a 25 años & 1.43 & $0.39 ; 4.95$ & 0.521 \\
Ocupación: Ama de casa & 2.43 & $0.74 ; 7.93$ & 0.092 \\
Tener Pareja & 1.73 & $0.49 ; 5.82$ & 0.317 \\
Escolaridad mayor o igual a 5 años & 1.32 & $0.41 ; 4.07$ & 0.589 \\
Ingresos de 1 o más SMMLV* & 0.43 & $0.12 ; 1.59$ & 0.146 \\
\hline \hline
\end{tabular}

${ }^{ \pm}$LME: Lactancia Materna Exclusiva

* SMMLV: Salario Mínimo Mensual Legal Vigente

${ }^{\dagger}$ Prueba $C h i^{2}$

\section{DISCUSIÓN}

\section{Conocimientos y creencias respecto a la lactancia materna}

Identificar los conocimientos y creencias que posee la población es importante para desarrollar estrategias que contribuyan a una adecuada salud y nutrición infantil. Uno de los factores que contribuye al éxito de la lactancia materna es la información que la madre recibe sobre sus beneficios y técnicas, lo cual propicia la confianza y seguridad de las mujeres para amamantar a sus hijos (10). El conocimiento de los beneficios de la LM, está asociado con la iniciación temprana y la duración de la LM y la iniciación tardía de fórmulas lácteas (14). Estudios nacionales muestran que las madres consideran que la LM es beneficiosa para el niño (15) y resaltan los aspectos inmunológicos y de salud (16). En esta investigación la mayoría de las madres tiene esa percepción. Los programas de capacitación enfatizan en los beneficios nutricionales de la LM y en la importancia del calostro, por su contenido de factores inmunitarios, proteínas, minerales y carotenoides. Un 63,5\% de las madres que participaron en este estudio conoc ían la importancia nutricional del calostro, porcentaje un poco mayor al reportado en otros estudios nacionales (16).

\section{Prevalencia de lactancia materna}

En Colombia, en el año 1995 se encontró que un 94,5\% de los niños menores de 5 años habían sido amamantados alguna vez, de ellos 50,5\% durante la primera hora y $81,3 \%$ durante las primeras 24 horas (17). En el año 2000 el 95,5\% de la población infantil había recibido LM, 61\% durante la primera hora, y $87 \%$ durante las primeras 24 horas (18), mientras en el año 2005 la proporción de niños amamantados fue de $97,1 \%$. 
En México la encuesta nacional de 1999, encontró que la proporción de niños amamantados alguna vez fue de $92,3 \%$, la prevalencia de LME en menores de 4 meses fue de 25,7\% y en menores de 6 meses de 20,3\% (19). En 1992 un estudio colombiano mostró que un 2,6\% nunca amamantó (16), similar a lo encontrado en la Encuesta Nacional colombiana del año 2005 (2,9\%) (11). En esta investigación la prevalencia reportada de LM hasta los doce meses fue de 20\% y el promedio de LME fue 5,7 meses.

\section{Duración y mantenimiento de LM y LME}

El reto de las políticas de LM, consiste en lograr no sólo un aumento de la población infantil que reciba alguna vez la LM, sino lograr mantenerla, lo cual es un desafío también para las familias, ya que existen diversos factores causales que impiden que este propósito se lleve a cabo. Las investigaciones muestran que la duración de LME se asocia al conocimiento de la LM, con la edad y las horas de trabajo de la madre. (20). Otros estudios recomiendan la implementación de programas que aumenten la aceptabilidad social de la LM y apoyen por largos periodos a la madre (21). La Organización Panamericana de la Salud (OPS) y el plan decenal 1998-2008 para la promoción y apoyo a la LM del Ministerio de Salud de Colombia, tiene como objetivo que la LME tenga una duración de seis meses, y que la LM continúe hasta los dos años (4). En Colombia, la duración promedio de LME en el año 2005 fue de 2,2 meses y la duración total promedio de LM de 14,9 meses. En México la duración promedio de LM es de 9 meses (19). Un estudio realizado en Cali, mostró que la duración es muy corta ya que a los 6 meses de vida, sólo un 1,6\% de los menores continuaban recibiendo LME (15). En Sri Lanka, las proporciones de amamantamiento exclusivo a los 4 y 6 meses fueron $61.6 \%$ y $15.5 \%$ respectivamente, el año 2006 (22).

Un factor favorecedor de la producción láctea es la succión; en las participantes de este estudio este factor es reconocido solamente por el 7,3\%, ellas aducen que el consumo de líquidos es el más importante; una quinta parte de las mujeres reconocen la alimentación adecuada como importante, situación similar a lo encontrado en otros estudios (16). En población procedente de Camboya éste fue el único factor reconocido como importante para la producción de LM (23).

Otro factor importante es el apoyo familiar especialmente el del esposo, y el apoyo social de amigos o de grupos organizados de mujeres que dan apoyo madre a madre para la lactancia y la crianza. En el presente trabajo cuatro quintas partes de las mujeres con hijos recibieron apoyo durante la LM, una tercera parte provenía del esposo y la familia, de igual manera a lo reportado por otro estudio nacional (16). 
Vale la pena resaltar la importancia que tiene para la población estudiada la colaboración recibida por el personal de salud, la cual se presentó en igual proporción a la recibida por la familia. Otros estudios también reportaron como factores importantes para la continuación de la LM a la atención prenatal y las prácticas hospitalarias de LM (24). La educación ofrecida en los programas del sector salud incide en la motivación de las madres hacia la LM; los estudios muestran que las intenciones prenatales y las experiencias de amamantamiento inciden en la iniciación o suspensión temprana de LM (25). Otro apoyo importante es el de los amigos, casi la mitad de la ayuda ofrecida fue dada por estas personas. Algunas madres lactantes manifestaron el placer que les ofrece compartir la experiencia con otras mujeres, enseñarles y apoyarlas, y constituirse como un apoyo y recuerdan la experiencia como gratificante (10). Cuando las madres encuestadas tienen problemas acuden a las madres, abuelas y al médico y además ellas consideran que los programas educativos son importantes para promocionar la LM (10).

Una estrategia para favorecer el mantenimiento de la LM, a pesar de los compromisos fuera del hogar, es la extracción de la leche, en este estudio se encontró que esta práctica se realiza en más del $80 \%$ de mujeres que han tenido hijos.

\section{Factores asociados a la sus pensión de la lactancia materna}

Las mujeres que participaron en este estudio consideraron que la edad apropiada para la ablactación es a los 7,8 meses, similar a lo encontrado en otro estudio nacional. (10). En la encuesta nacional de Colombia del año 2005, la edad promedio de inicio de la ablactación fue de 5 meses.

En Colombia las principales causas de suspensión de LM en menores de tres años identificadas en el año 2005 fueron la edad del niño $(29,3 \%)$, rechazo del niño $(22,4 \%)$, insuficiente producción de leche de la madre $(19,7 \%)$, trabajo de la madre $(8,5 \%)$ y enfermedades de la madre $(6,5 \%)$. En los primeros tres meses las causas principales son: no tener leche $(60,5 \%)$ y problemas del pezón (10,5\%) (11).

En otros estudios nacionales, se encontró que a un $65 \%$ de los niños se les suspendió la LM a los 6 meses, y los motivos fueron la poca producción láctea en el 19\% y el rechazo por parte de los niños en el $24 \%$; éstas razones también fueron reportadas en otro estudio (23).

Se ha encontrado que la preocupación de la madre por la producción insuficiente de leche es un factor que incide en la corta duración de la LM (14). Otras causas reportadas en el presente estudio fueron: la enfermedad de la madre 15\%, el trabajo $9 \%$ y la enfermedad del niño $2 \%$. El trabajo fue la causa de suspensión de la lactancia materna en otros estudios nacionales e internacionales (26), sin embargo el factor laboral que incide en la práctica de LM es la ausencia de facilidades para lactar (27). 
Otros factores son el nivel educativo de la madre, el nivel socioeconómico de las familias y asistencia a control prenatal $(28,29)$. En Sri Lanka la cesación temprana de LM estuvo asociada con el bajo nivel educativo y el desempleo en la madre (22). La inclusión de otros alimentos, incluido las fórmulas lácteas también es una causa determinante en el abandono de la LM. (7, 29).

Respecto al uso del biberón, según la encuesta nacional de nutrición de Colombia en el año 2005, un 65,0\% de los menores de un año lo usan (11); similar a lo reportado en Sri Lanka, donde la proporción fue de $61.6 \%$ (22).

\section{CONCLUSIONES}

Los resultados de este estudio permitieron identificar los aspectos críticos en cuanto al manejo de las dificultades que se pueden presentar durante la lactancia materna y los aspectos que favorecen el abandono de esta práctica. Estos hallazgos hicieron parte del diagnóstico de la situación de salud realizado por el departamento de Salud Pública y las escuelas de Nutrición y Dietética, Trabajo Social y Enfermería de la Universidad Industrial de Santander y permitieron implementar estrategias de intervención para mitigar la problemática encontrada. Este estudio generó un alto impacto en la población de estudio dado que permitió la formulación y la ejecución de un programa de Educación Nutricional que fomentó la práctica de la lactancia materna en las mujeres residentes en este asentamiento, acciones que se llevaron a cabo de manera conjunta con el Instituto Colombiano de Bienestar Familiar, ICBF, (programa estatal que tiene como propósito prevenir el deterioro del estado nutricional de las mujeres gestantes, madres lactantes y menores de siete años) y que justificaron la creación de un Hogar FAMI (Familia, Mujer e Infancia), por parte del ICBF, en este asentamiento.

\section{RESUMEN}

Objetivo: Identificar los conocimientos, actitudes, prácticas relacionados con la Lactancia Materna (LM) en mujeres en edad fértil residentes del asentamiento las Marías del Municipio de Girón en el año 2005. Sujetos y métodos: Se realizó un corte transversal descriptivo con 96 mujeres en edad fértil del asentamiento las Marías, Girón, 2005. Se aplicó una encuesta tipo Conocimientos, Actitudes y Prácticas (CAP). Los datos fueron digitados y validados en Epi Info 6,04 y analizados en Stata 8,2. Para analizar las variables continuas, se utilizaron medidas de tendencia central y de variabilidad; las variables categóricas fueron expresadas como proporciones. Resultados: El 78,1\% de las participantes había tenido hijos. Sólo el 13\% tenía conocimientos sobre la forma correcta de amamantar. Un $49 \%$ no conocía los métodos de conservación de la leche materna. El 69,8\% de las participantes tiene actitud de apoyo cuando ve a una familiar lactando. El tiempo promedio de lactancia materna fue 5,7 meses. El alimento con el que más inician la alimentación complementaria es el caldo (88\%). Conclusiones: se presentaron proporciones considerables de mujeres que dieron LM durante menos de seis meses, que iniciaron la alimentación complementaria antes de los 6 meses y que suspendieron la LM cuando el hijo lactante sufrió alguna enfermedad.

Palabras claves: lactancia materna; conocimientos; actitudes; prácticas; población vulnerable. 


\section{BIBLIOGRAFIA}

1. Valdés, Soler Amanda; Rozo, Mariño Adriana. Guías alimentarías para gestantes y madres en lactancia. Ministerio de la protección social, Instituto Colombiano de Bienestar Familiar, Fundación Colombiana para la nutrición Infantil (NUTRIR). Santa Fe de Bogotá, D.C. 2004.

2. WHO -UNICEF-USAID. Innocent declaration on protection, promotion an support of breastfeeding. New York: WHO- UNICEF- USAID; 1990.

3. Instituto Proinapsa, Universidad Industrial de Santander. Alcaldía de Bucaramanga, Secretaria de Salud y del ambiente. Contactos cercanos en la vida láctea. Manual para el Desarrollo de la Iniciativa de Instituciones Amigas de la Mujer y la Infancia. Bucaramanga, 2001.

4. Minsalud- UNICEF. Plan decenal para la promoción, protección y apoyo a la Lactancia Materna 1998-2008. Ministerio de Salud y UNICEF. Bogotá 1998.

5. Marquis GS, Habicht JP, Lannata CF, Black RE, Rasmussen KM. Breast milk of animal-product foods improve linear growth of Peruvian toddlers consuming marginal diets. Am $\mathbf{J}$ Clin Nutr 1997;66:1102-1109.

6. López-Alarcón M, Villalpando S, Fajardo A. Breast-feeding lowers the frequency and duration of acute respiratory infection and diarrea in infants under six months af age. J Nutr 1997; 127:436-443.

7. UNICEF. Por una niñez bien nutrida:Comunicación para la Acción. Bogota, Colombia. 2004.

8. Institute of Medicine, Subcommittee on Nutrition During Lactation, Comité on Nutritional Status during Pregnancy and Lactation, Food and Nutrition Board. Nutrittion during Lactation. Washington, DC. National Academy Press, 1991.

9. Bowman, Bárbara A.; Russell Robert M. Conocimientos actuales sobre Nutrición. Octava edición. Publicación Científica y Técnica No 592. OPS-OMS. Washington 2003. pp 441-454.

10. Ochoa G, Carreño SP. Conocimientos, saberes y prácticas sobre lactancia materna. Estudio de línea de base. DABS 2003. Alcadía Mayor de Bogotá. Bogota. 2003.

11. ICBF, Profamilia. Instituto Nacional de Salud, Universidad de Antioquia, OPS. Encuesta nacional de la situación nutricional en Colombia. 2005. Bogota. Colombia.

12. OPS. Universidad de Antioquia. Serie Salud y desplazamiento en Colombia: Comparación de la situación de salud entre población en situación de desplazamiento y receptora, en seis ciudades 2002-2003. Modulo 1.

13. PMA, OPS, Comisión Europea de Ayuda Humanitaria. Estado nutricional, de alimentación y condiciones de salud de la población desplazada por la violencia en seis subregiones de Colombia. Unión Temporal de Econometría SA. SEI SA. Bogotá. Colombia. 2005.

14. McCann M, Baydar N, Williams R . Breastfeeding Attitudes and Reported Problems in a National Sample of WIC Participants. Journal of Hum Lactation. 2007; 23: 314-324.

15. Cabrera GA, Mateus, JC, Girón SL. Duración de la lactancia exclusiva en Cali, Colombia 2003. Colomb. Med. 2004; 35 (3):132-138.

16. Carrasquilla G, Osorno J, Paredes B, Sota A, Vásquez C. Lactancia materna en zonas marginadas de grandes ciudades Colombianas. Fundación FES, Fundación Santa Fe de Bogotá. 1992. 
17. Profamilia. Encuesta Nacional de Demografía y Salud. 1995. Bogota. Colombia.

18. Profamilia. Salud Sexual y Reproductiva. Encuesta Nacional de Demografía y Salud. 2000. Bogota. Colombia.

19. Gonzáles-Cossio T, Moreno-Macía s H, Rivera JA, Villalpando S, Shamah-Levy T. Monterrubio EA, Hernández-Garduño A. Prácticas de lactancia en México: resultados de la segunda Encuesta Nacional de Nutrición 1999. Salud Pública Mex 2003; 45 supl 4:5477-5489

20. Gijsbers B, Mesters I, Knottnerus JA, van Schayck CP. Factors associated with the duration of exclusive breast-feeding in asthmatic families. Health Education Research Advance Access published online on April 5, 2007. Health Education Research, doi:10.1093/her/cym013.

21. Rempel LA. Factors Influencing the Breastfeeding Decisions of Long-term Breastfeeders. Journal of Human Lactation, 8 2004; vol. 20: pp. 306 - 318.

22. Agampodi S. Agampodi T, Kankanamge U, Piyaseeli D. Breastfeeding practices in a public health field practice area in Sri Lanka: a survival analysis. International Breastfeeding Journal. 2007; $2: 13$

23. Straub B, Melvin C, Labbok M. A descriptive study of Cambodian refugee infant feeding practices in the United States. Internat Breastfeeding J 2008; 3:2.

24. Estevez MD, Martell D, Medina R, García E, Saavedra P. Factores relacionados con el abandono de la lactancia materna. An Es Pediatr 2002; 56(2): 144-150.

25. Di Girolamo A, Thompson N, Martorell R , Fein S , Grummer-Strawn L. Intention or Experience? Predictors of Continued Breastfeeding Health Educ Behav.2005; 32: 208-226.

26. Roe B, Whittington L, Fein Sb, Teisl MF. Is there a competition between breast-feeding and maternal employment?. Demography 1999;36:157-171.

27. Navarro-Estrella M, Duque-López MX, Trejo y Pérez JA. Factores que influyen en el abandono temprano de la lactancia por mujeres trabajadoras. Salud Publica Mex 2003;45:276-284.

28. Vandale-Toney S, Rivera-Pasquel ME, Kageyama-Escobar ML, Tirado-Gómez LL, López_Cervantes M. Lactancia materna, destete, ablactación: una encuesta en comunidades rurales de México. Salud Pública Mex 1997; 39(5): 412-419.

29. Bautista L. Factores asociados al inicio de la lactancia materna en mujeres dominicanas. Rev Panan Salud Pública 1997; 1(3):200-206. 